\title{
On quantum approach to modeling of plasmon photovoltaic effect
}

\author{
Kluczyk, Katarzyna; David, Christin; Jacak, Witold Aleksander
}

Published in:

Journal of the Optical Society of America B-optical Physics

Link to article, DOI:

10.1364/JOSAB.34.002115

Publication date:

2017

Document Version

Peer reviewed version

Link back to DTU Orbit

Citation (APA):

Kluczyk, K., David, C., \& Jacak, W. A. (2017). On quantum approach to modeling of plasmon photovoltaic effect. Journal of the Optical Society of America B-optical Physics, 34(10), 2115-2127.

https://doi.org/10.1364/JOSAB.34.002115

\section{General rights}

Copyright and moral rights for the publications made accessible in the public portal are retained by the authors and/or other copyright owners and it is a condition of accessing publications that users recognise and abide by the legal requirements associated with these rights.

- Users may download and print one copy of any publication from the public portal for the purpose of private study or research.

- You may not further distribute the material or use it for any profit-making activity or commercial gain

- You may freely distribute the URL identifying the publication in the public portal

If you believe that this document breaches copyright please contact us providing details, and we will remove access to the work immediately and investigate your claim. 


\title{
On quantum approach to modeling of plasmon photovoltaic effect
}

\author{
Katarzyna KluczyK ${ }^{1,}$, Christin David ${ }^{2}$, and Witold A. JacaK ${ }^{1}$ \\ ${ }^{1}$ Department of Quantum Technologies, Wrocław University of Science and Technology, Poland \\ ${ }^{2}$ Department of Photonic Engineering, DTU, Denmark \\ *Corresponding author: katarzyna.kluczyk@pwr.edu.pl
}

Compiled July 26, 2017

\begin{abstract}
Surface plasmons in metallic nanostructures including metallically nano-modified solar cells are conventionally studied and modeled by application of the Mie approach to plasmons or by the finite element solution of differential Maxwell equations with imposed boundary and material constraints (e.g., upon commercial Comsol software-system). Both approaches are essentially classical ones and neglect quantum particularities related to plasmon excitations in metallic components. We demonstrate that these quantum plasmon effects are of crucial importance especially in theoretical simulations of plasmon-aided photovoltaic phenomena. Quantum corrections considerably improve both the Mie and Comsol approches in this case. We present the semiclassical random phase approximation description of plasmons in metallic nanoparticle and apply the quantum Fermi golden rule scheme to assess the sun-light energy transfer to the semiconductor solar cell mediated by surface plasmons in metallic nanoparticles deposited on the top of the battery. In addition, short-ranged electron-electron interaction in metals is discussed in the framework of the semiclassical hydrodynamic model. The significance of the related quantum corrections are illustrated by the quantumly improved Comsol simulations. () 2017 Optical Society of America
\end{abstract}

OCIS codes: (160.3900) Metals; (160.4236) Nanomaterials;(260.3910) Metal optics;(350.4990) Solar energy.

http://dx.doi.org/10.1364/ao.XX.XXXXXX

\section{INTRODUCTION}

In many photodiode setups and photo-cell systems a significant increase in efficiency has been observed due to mediation of plasmons in the process of sun-light energy harvesting [1-8]. Even though an enhancement of the photo-current in a laboratory photodiode setup reaches $100 \%$ increase, the overall gain in realistic solar cells is much more modest because the photo-effect aided by plasmons is only one element of a long series of factors deciding on the final efficiency of a cell. Recently, an efficiency increase up to several percent has been observed in multi-crystalline silicon cells covered with not densely distributed gold or silver nanoparticles with radius of several tens of nanometers [9]. It must be emphasized that such an increase is very promising in view of other photovoltaic technology, like thin film solar cells, organic plastic cells or newly developed perovskite cells, where an increase even by a few percent would be important to obtain industrially competitive cell designs [4, 7, 10-18].

Such encouraging prospects in experiments cause also a rapid increase of theoretical studies of the related physical effects and of numerical modeling and simulations of metallically nanomodified solar batteries. Especially popular are numerical methods for study of surface plasmons in metallic nanoparticles utiliz- ing the finite element method for solution of Maxwell differential equations with geometry and material constraints imposed on the propagating electromagnetic (e-m) wave corresponding to a sun-light beam incident on the photoactive surface of a substrate semiconductor with metallic component deposited on the top. This approach finds a correspondence with the classical analytical solution of the problem of scattering of the incident e-m plane wave on the spherical metallic body $[19,20]$. The solution of the related Fresnel equations both by Mie approach or by the finite element method utilize as a prerequisite the dielectric function for metals, usually in the simplified form upon the Drude approximation [20]. This appears to be sufficient to display surface plasmon modes for absorption of incident light with frequencies much lower than the bulk plasmon frequency [21], $\omega_{p}=\sqrt{\frac{e^{2} n}{\varepsilon_{0} m}}$. The surface plasmon modes are dependent on multipole number corresponding to the decomposition of plasma oscillations in spherical functions adjusted to the spherical system geometry. The lowest such frequency, $\omega_{1}=\frac{\omega_{p}}{\sqrt{3}}$, is usually called Mie frequency [19]. It is worth noting that the surface plasmon frequencies are determined by the geometry type constraints imposed on the completely classical Maxwell equa- 
tions. The information on microscopy of the plasma dynamics in metallic sphere is packed into phenomenological parameters, $\omega_{p}$ and $\gamma$, modeling the Drude-Lorentz dielectric function for free electrons [20],

$$
\varepsilon=1-\frac{\omega_{p}^{2}}{\omega^{2}+i \gamma \omega},
$$

where $\omega_{p}$-bulk plasmon frequency, $\gamma$-damping rate for plasmons. Hence, in such approaches is no room for quantum details unless the dielectric function of the metallic nanosphere is more precisely identified. We will inspect this way for quantum corrections to the e-m response of metallic nanoparticle especially in view of better simulation of metallically modified solar cells upon the commercial system Comsol utilizing the finite element method for Maxwell equation solution. Furthermore, we discuss the modification of Mie coefficients to include spatial dispersion effects of electrons interacting with each other over short distances. Such nonlocal effects are introduced in the framework of the hydrodynamic model [22-26].

\section{SURFACE PLASMONS MEDIATE ENERGY TRANS- FER IN METALLICALLY NANO-MODIFIED SOLAR CELLS}

Plasmons are collective excitations of the electron liquid in metals [21] with energy similar to Fermi energy, $\hbar \omega_{p} \sim \epsilon_{F}\left(\epsilon_{F}\right.$ is the Fermi energy in metal, typically of order of $7-8 \mathrm{eV})$. Plasmons do not interact with low energy excitations near the Fermi surface (single electrons), but can be excited by photons with adjusted energy (UV in bulk and visible light in nanoparticles [20]). Many approaches were used to describe plasmons. The random phase approximation (RPA) [21] occurred to be very convenient to describe volume plasmons in bulk and then this approach has been accommodated to metallic finite systems [27-30]. Other methods utilized the Kohn-Sham approach in numerical version of related calculus called LDA (Local Density Approximation) and its time-dependent version (TDLDA) [27-29, 31, 32]. In metal clusters these numerical quasi-exact studies are, however, effective only for ultra-small clusters, up to approximately 300 electrons, i.e., $2-3 \mathrm{~nm}$ of cluster size. For larger clusters, the random phase approximation (RPA) [30] appears to be efficient and sufficiently accurate. In these studies the jellium model is usually applied to avoid the inertial heavy ion crystal lattice dynamics [27, 28, 31]. The origin of high-energy of plasmons (similar as the Fermi energy) is the Coulomb interaction of electrons with jellium and the coherent participation of all electrons in plasma oscillations in contrast to single-particle excitations (Landau quasiparticles) close to the Fermi surface [21, 30, 33, 34]. In the case of bulk metal only volume plasmons are possible [21], whereas in finite metallic systems also surface plasmon modes can be excited besides volume ones. The surface plasmons correspond to the translational type mode of coherent oscillations of all electrons with respect to the jellium, whereas the volume plasmons are collective compressional-type osillations of electrons. The former result in the uncompensated charge of fluctuations only on the surfaces of the finite system, whereas the lattercompressional fluctuations still possesses their volume character [30]. Interestingly, the frequencies of volume modes in metallic nanoparticles are larger than $\omega_{p}$, whereas the frequencies of the surface modes are smaller than $\omega_{p}$ [30]. For photovoltaic applications important are nanoparticles of noble metals (Au, Ag and also $\mathrm{Cu}$ ) for which surface plasmon frequencies conveniently enter the visible part of the e-m spectrum.
Surface plasmon frequencies appear to be strongly size dependent. In ultra-small clusters this dependence is caused mostly by so-called spill-out of electrons beyond the rim of the jellium, which causes a dillution of the electron density and reduces plasmon energy [28]. The spill-out effect is, however, negligible for larger clusters (with radius larger than appr. $5 \mathrm{~nm}$ ). In the case of large metallic nanospheres it has been proved that the strong size dependence of plasmon energy is governed by irradiation effects (Lorentz friction) [30, 35-37].

The strong plasmon irradiation from metallic nanoparticles makes plasmons usable for solar cell applications as of high efficiency energy transmitters [2, 3, 5, 38-41], because due to absorption-emission symmetry the large metallic nanoparticles absorb sun-light energy with exceptionally high efficiency $[42,43]$ and then may transfer the collected energy in plasmon oscillation to semiconductor substrate via coupling of plasmons with semiconductor band electrons. This coupling appears to be much stronger than coupling of free space irradiation with band electrons $[30,36]$ and the sun-light energy may flow to the substrate semiconductor highly beyond the efficiency of the ordinary photo-effect when is mediated by plasmons. The efficiency gain in plasmon mediated photo-effect reaches even $200-300 \%$ increase beyond the ordinary photo-effect $[30,36]$. It contributes to an overall gain of a solar cell efficiency in much modest way because other process contribute and the plasmon mediated photo-effect is only the initial stage of the series of furthers steps- 6 percent eventual increase has been demonstrated in conventional Si-multi-crystalline cell [9].

The plasmonic-induced efficiency enhancement of the photoeffect is related to the trade-off between two opposite mechanisms [30, 36]. An enhancement of the electric field near the metallic nanoparticle due to growing amplitude of the surface plasmon oscillations is proportional to $a^{3}$ ( $a$ is the metallic nanosphere radius) because all electrons participate in the translational-type oscillations of surface plasmons. This local concentration of the electric field strengthens coupling to band electrons in the substrate semiconductors, and prefers larger $a$. Nevertheless, the other important effect favors smaller $a$-it is related to the contribution of all indirect inter-band electron transitions in the semiconductor substrate induced by plasmons. For local coupling with plasmons in a nanoparticle, the translation invariance is violated and the momentum conservation does not hold in contrary to the ordinary photo-effect being translational invariant. Reducing of the momentum conservation constraint apparently prefers thus the smaller nanoparticle when the larger amount of indirect inter-band transitions contributes [30, 36]. The related size-competition gives rise to optimize the size of nanoparticles for photo-voltaic applications. The details of this efficiency trade-off are presented below.

\section{A. Plasmons in large metallic nanospheres}

Plasmons in metallic clusters were intensively studied since 80. of the last century by application of the Kohn-Sham approach in a numerical manner. The related precise quantum study called as LDA (Local Density Approximation) and its time-dependent generalization TDLDA [27-29, 31, 32] were, however, ranged to ca. 300 electrons as sharply limited by rapid growth of required computation resources with rising number of electrons. Hence, the method LDA (TDLDA) appeared useful rather for ultra-small metallic nanoparticles with size of order of $1-3 \mathrm{~nm}$. Such small nanoparticles exhibit, however, a different collective behavior than larger clusters. In the case of the extreme small clusters (of size $1-3 \mathrm{~nm}$ ) all the physics is dominated by surface 
effects including the pronounced in this scale a spill-out of electrons beyond the jellium rim. The dilution of electrons caused by the spill-out is the most important factor shifting the surface plasmon resonance in such small particles. The resulting red-shift of surface plasmon frequency has been studied experimentally in ultra-small clusters of $\mathrm{K}$ and $\mathrm{Au}$ [28]. Nevertheless, in larger metallic nanoparticles with radius greater than $5 \mathrm{~nm}$ all effects induced by spill-out are negligible as quickly lowering with the radius growth. In this size scale the so-called Landau damping of plasmons (i.e., the decay of plasmons for high energy particlehole pairs) is also small and practically negligible [28]. Moreover, in larger nanoparticles (with radius exceeding $10 \mathrm{~nm}$, for noble metals) quite different mechanism of plasmon damping occurred to be important $[30,37]$. The latter corresponds to the irradiation of energy of plasmons (the synchrotron-type effect), intensity of which grows with number of electrons in the nanoparticle, $\sim a^{3}$ ( $a$ is the nanoparticle radius). The resulting strong damping of plasmons in nanoparticles with $a \in(10,100) \mathrm{nm}$ dominates the plasmon physics in this scale. To account a quantum behavior of plasmons in this scale the random phase approximation (RPA) $[21,30]$ occurred to be very convenient. The RPA theory has been proposed originally by Pines and Bohm [21] to describe volume plasmons in bulk and was next accommodated to the metallic finite systems [27-30]. This methods appears to be effective and sufficiently accurate in the size scale $a \sim 10-100 \mathrm{~nm}$, especially in view of the shortage of LDA (TDLDA) methods in this scale. Moreover, the RPA approach allowed for an analytical definition of plasmon resonances and their damping [30,37] simplifying further applications.

The dynamic equations for plasmons in a metallic nanosphere have been derived in [30] upon the RPA formulation via utilization of the Heisenberg dynamic equation for time dependent operator of local density of electrons [21]. According to this theory (for details of derivation cf. [30]), the electron density fluctuations can be represented for the volume and the surface modes, respectively,

$$
\begin{array}{ll}
\delta \tilde{\rho}_{1}(\mathbf{r}, t)=n_{e} F(\mathbf{r}, t), & \text { for } r<a, \\
\delta \tilde{\rho}_{2}(\mathbf{r}, t)=\sigma(\Omega, t) \delta(r+\epsilon-a), \epsilon=0+, & \text { for } r \geq a,(r \rightarrow a+),
\end{array}
$$

where $n_{e}=\frac{N_{e}}{V}$ is equilibrium density of electrons.

For the spherical symmetry of the nanoparticle one can find the solution of the dynamic equations written in the above notation [30],

$$
F(\mathbf{r}, t)=\sum_{l=1}^{\infty} \sum_{m=-l}^{l} \sum_{n=1}^{\infty} A_{l m n} j_{l}\left(k_{n l} r\right) Y_{l m}(\Omega) \sin \left(\omega_{n l} t\right)
$$

and

$$
\begin{aligned}
\sigma(\Omega, t)=\sum_{l=1}^{\infty} \sum_{m=-l}^{l} \frac{B_{l m}}{a^{2}} Y_{l m}(\Omega) \sin \left(\omega_{0 l} t\right) \\
+\sum_{l=1}^{\infty} \sum_{m=-l}^{l} \sum_{n=1}^{\infty} A_{l m n} \frac{(l+1) \omega_{p}^{2}}{l \omega_{p}^{2}-(2 l+1) \omega_{n l}^{2}} Y_{l m}(\Omega) n_{e} \\
\quad \times \int_{0}^{a} d r_{1} \frac{r_{1}^{l+2}}{a^{l+2}} j_{l}\left(k_{n l} r_{1}\right) \sin \left(\omega_{n l} t\right),
\end{aligned}
$$

where $j_{l}(\xi)=\sqrt{\frac{\pi}{2 \xi}} I_{l+1 / 2}(\xi)$ is the spherical Bessel functions, and $Y_{l m}(\Omega)$ is the spherical functions, associated with the spherical symmetry of the metallic particle. The frequencies $\omega_{n l}=\omega_{p} \sqrt{1+\frac{x_{n l}^{2}}{k_{T}^{2} a^{2}}}$ define frequencies of the volume plasmon self-oscillations, where $k_{T}=\sqrt{\frac{6 n_{e} e^{2}}{\epsilon_{F}}}$ is the Thomas-Fermi length ( $\epsilon_{F}$ is the Fermi energy), the $x_{n l}$ are the nodes of the Bessel function $j_{l}(\xi), k_{n l}=x_{n l} / a$. The frequency $\omega_{0 l}=\omega_{p} \sqrt{\frac{l}{2 l+1}}$ is frequency of $l$-multipole mode of surface self-oscillations (surface plasmons). The function $F(\mathbf{r}, t)$ describes the volume plasmon oscillations, whereas $\sigma(\Omega, t)$ the surface plasmon oscillations. In the formula (4) the first term corresponds to surface selfoscillations, whereas the second term describes the surface oscillations induced by the volume plasmons. The frequencies of the surface self-oscillations are, $\omega_{0 l}=\omega_{p} \sqrt{\frac{l}{2 l+1}}$., which for $l=1$ gives the dipole-type surface oscillation frequency originally found by Mie [19], $\omega_{01}=\omega_{p} / \sqrt{3}$ (we denote it as $\omega_{1}=\omega_{01}$ ).

\section{B. Damping of plasmons in large metallic nanoparticles in di- electric surroundings}

The damping of plasmons in metallic nanoparticles in dielectric surroundings is induced by the scattering of electrons on other electrons, on defects, on phonons and on nanoparticle boundaries [44] and by the Loretz friction [37]. The electron scattering can be described in common by the damping rate,

$$
\frac{1}{\tau_{0}} \simeq \frac{v_{F}}{2 \lambda_{B}}+\frac{C v_{F}}{2 a}
$$

where $a$ is the nanosphere radius, $v_{F}$ is the Fermi velocity in the metal, and $\lambda_{B}$ is the electron mean free path in the bulk, $C$ is a constant of order of unity and its particular value displays in a phenomenological manner the type of scattering of electrons on the edge of a nanoparticle (diffusive, reflective or intermediate). The first term in (5) evaluates all the scattering processes similar to those in the bulk, the second one displays scattering of electrons on the nanoparticle edge. Dissipation of energy of plasmons due to scattering of electrons hampers both volume and surface plasmons.

The Lorentz friction-induced plasmon damping can be accounted for by the solution of the RPA dynamic equation for plasmons in the nanoparticle. In the case of metallic nanoparticles with radius well smaller that the resonant wavelength of only the dipole $(l=1)$, surface plasmon oscillations contribute to the e-m response (to the sun-light illumination), because of dipole approximation constraint (for wavelength much larger than the nanoparticle size). The corresponding dipole of the plasmon oscillations attains the form [30],

$$
\mathbf{D}(t)=e \int d^{3} r \mathbf{r} \delta \rho(\mathbf{r}, t)=\frac{4 \pi}{3} e \mathbf{q}(t) a^{3} .
$$

and this dipole satisfies the following equation:

$$
\left[\frac{\partial^{2}}{\partial t^{2}}+\frac{2}{\tau_{0}} \frac{\partial}{\partial t}+\omega_{1}^{2}\right] \mathbf{q}(t)=\frac{e n_{e}}{m} \mathbf{E}(t),
$$

where $\mathbf{E}(t)$ is the electric field component of an incident light beam. The Lorentz friction which hampers the oscillations of a plasmon dipole has the form [45],

$$
\mathbf{E}_{L}=\frac{2}{3 c^{3}} \frac{\partial^{3} \mathbf{D}(t)}{\partial t^{3}}
$$

where $\mathbf{E}_{L}$ is an additional electric field braking the charge oscillations, $c$ is the light velocity and $\mathbf{D}(t)$ is the dipole-here of the 
surface plasmon oscillations. Due to Eq. (6), one can rewrite the above formula,

$$
\mathbf{E}_{L}=\frac{2 e}{3 c^{3}} \frac{4 \pi}{3} a^{3} \frac{\partial^{3} \mathbf{q}(t)}{\partial t^{3}} .
$$

The Lorentz friction field contributes the dynamic equation,

$$
\left[\frac{\partial^{2}}{\partial t^{2}}+\frac{2}{\tau_{0}} \frac{\partial}{\partial t}+\omega_{1}^{2}\right] \mathbf{q}(t)=\frac{e n_{e}}{m} \mathbf{E}(t)+\frac{2}{3 \omega_{1}}\left(\frac{\omega_{1} a}{c}\right)^{3} \frac{\partial^{3} \mathbf{q}(t)}{\partial t^{3}} .
$$

One can find the exact solution of Eq. (10) for the damping and self-frequency including Lorentz friction is as follows [37] (the exponents of solution $\sim e^{i \Omega t}$ for self-modes)

$$
\begin{aligned}
& \Omega_{1}=-\frac{i}{3 l}-\frac{i 2^{1 / 3}(1+6 l q)}{3 l \mathcal{A}}-\frac{i \mathcal{A}}{2^{1 / 3} 3 l}=i \alpha \in I m, \\
& \Omega_{2}=-\frac{i}{3 l}+\frac{i(1+i \sqrt{3})(1+6 l q)}{2^{2 / 3} 3 l \mathcal{A}}+\frac{i(1-i \sqrt{3}) \mathcal{A}}{2^{1 / 3} 6 l}=\omega+i \frac{1}{\tau}, \\
& \Omega_{3}=-\omega+i \frac{1}{\tau}=-\Omega_{2}^{*},
\end{aligned}
$$

where $\mathcal{A}=\left(\mathcal{B}+\sqrt{4(-1-6 l q)^{3}+\mathcal{B}^{2}}\right)^{1 / 3}, \mathcal{B}=2+27 l^{2}+18 l q$, $q=\frac{1}{\tau_{0} \omega_{1}}$ and $l=\frac{2}{3 \sqrt{\epsilon_{0}}}\left(\frac{a \omega_{p}}{c \sqrt{3}}\right)^{3}$. The functions $\omega$ and $\frac{1}{\tau}$ (in dimensionless units, i.e., divided by $\omega_{1}$ ) are plotted in Fig. 1 versus the nanosphere radius $a$. Noticeable is the strong deviation from the perturbatively obtained behavior, $\frac{1}{\tau} \sim a^{3}$, which is apparent for $a>30 \mathrm{~nm}[30,37]$.

The exact solution (11) improved the fitting with the experimental measurements of the resonance frequency of dipole surface plasmons [37].

\section{Damping of plasmons due to coupling to band electrons in semiconductor substrate}

If another electrical system is present in the vicinity of the nanosphere, the situation changes considerably. The coupling of plasmons with other charged system causes resonant energy transfer, which results in a huge increase in the plasmon damping. In the case in which the nanosphere is deposited on the surface of a semiconductor, the near-field coupling of dipole plasmons with the band electrons of the semiconductor creates the very efficient channel to energy transfer. The attenuation rate corresponding to this process can be found by the application of Fermi golden rule approach to near-field coupling of plasmons with electrons [30, 46].

The Fourier components of the electric $\left(\mathbf{E}_{\omega}\right)$ field induced at a distant point $\mathbf{R}$ by monochromatic plasmonic dipole oscillation at the origin, $\mathbf{D}=\mathbf{D}_{0} e^{-i \omega t}$, has the form, including the retardation effect [45],

$$
\begin{aligned}
\mathbf{E}_{\omega}=\frac{1}{\varepsilon}\left\{\mathbf{D}_{0}(\right. & \left.\frac{k^{2}}{R}+\frac{i k}{R^{2}}-\frac{1}{R^{3}}\right) \\
& \left.+\hat{\mathbf{n}}\left(\hat{\mathbf{n}} \cdot \mathbf{D}_{0}\right)\left(-\frac{k^{2}}{R}-\frac{3 i k}{R^{2}}+\frac{3}{R^{3}}\right)\right\} e^{i k R},
\end{aligned}
$$

where we use the following notation for the retarded argument: $i \omega\left(t-\frac{R}{c}\right)=i \omega t-i k R$ and $\hat{\mathbf{n}}=\frac{\mathbf{R}}{R}$. The contributions with denominators of $R^{3}, R^{2}$, and $R$ are referred to as the near-, mediumand far-field zones of the dipole radiation, respectively. For interactions with the adjacent layer of the semiconductor, the nearfield zone prevails. Hence, one can neglect the terms containing $\frac{1}{R}$ and $\frac{1}{R^{2}}$. For the near field zone, one can obtain, $\mathbf{B}_{\omega}=0$ and $\mathbf{E}_{\omega}=\frac{1}{\varepsilon R^{3}}\left[3 \hat{\mathbf{n}}\left(\hat{\mathbf{n}} \cdot \mathbf{D}_{0}\right)-\mathbf{D}_{0}\right]$, which corresponds to the dipole electric field. The magnetic field is not important in the near-field

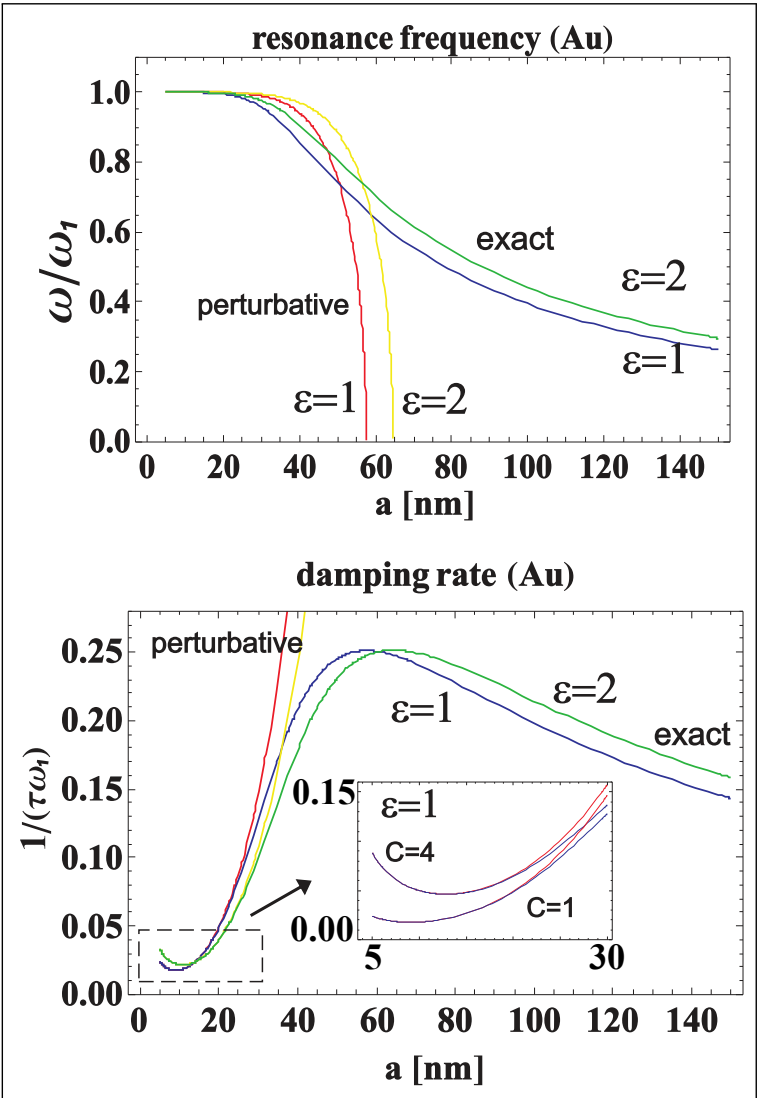

Fig. 1. The real and imaginary parts of the exact oscillating solution of Eq. (10), i.e., the resonance frequency and the damping rate given by Eq. (11) are plotted as the functions of the nanosphere radius $a$ and for two distinct dielectric surrounding media (vacuum and water); the lines corresponding to the perturbative solutions are presented for comparison; in the inset an impact of $C$ constant in Eq. (5) is illustrated.

zone [45]. The related potential for the near-field interaction of the Mie-type surface plasmons with the band electrons can be written as follows, [45]:

$$
\begin{aligned}
& w=e \psi(\mathbf{R}, t)=\frac{e}{\varepsilon R^{2}} \hat{\mathbf{n}} \cdot \mathbf{D}_{0} \sin (\omega t+\alpha)=w^{+} e^{i \omega t}+w^{-} e^{-i \omega t}, \\
& w^{+}=\left(w^{-}\right)^{*}=\frac{e}{\varepsilon R^{2}} \frac{e^{i \alpha}}{2 i} \hat{\mathbf{n}} \cdot \mathbf{D}_{0},
\end{aligned}
$$

$w^{+}$and $w^{-}$correspond to emission and absorption, respectively ( $\hat{\mathbf{n}}=\frac{\mathbf{R}}{R}$, and $\mathbf{D}_{0}$ is the plasmon dipole amplitude).

The semiconductor band system we model here in the simplest form of single-band parabolic effective mass approximation. According to the Fermi golden rule, the inter-band transition probability is given by the expression,

$$
w\left(\mathbf{k}_{1}, \mathbf{k}_{2}\right)=\frac{2 \pi}{\hbar}\left|<\mathbf{k}_{1}\right| w^{+}\left|\mathbf{k}_{2}>\right|^{2} \delta\left(E_{p}\left(\mathbf{k}_{1}\right)-E_{n}\left(\mathbf{k}_{2}\right)+\hbar \omega\right),
$$

where the Bloch states in the conduction and valence bands are assumed to be plane waves (to simplify calculations):

$$
\begin{aligned}
& \Psi_{\mathbf{k}_{1}}=\frac{1}{(2 \pi)^{3 / 2}} e^{i \mathbf{k}_{1} \cdot \mathbf{R}-i E_{p}\left(\mathbf{k}_{1}\right) t / \hbar,}, \\
& \Psi_{\mathbf{k}_{2}}=\frac{1}{(2 \pi)^{3 / 2}} e^{i \mathbf{k}_{2} \cdot \mathbf{R}-i E_{n}\left(\mathbf{k}_{2}\right) t / \hbar,}, \\
& E_{p}\left(\mathbf{k}_{1}\right)=-\frac{\hbar^{2} \mathbf{k}_{1}^{2}}{2 m_{p}^{*}}-E_{g}, E_{n}\left(\mathbf{k}_{2}\right)=\frac{\hbar^{2} \mathbf{k}_{2}^{2}}{2 m_{n}^{*}},
\end{aligned}
$$


where the indices $n$ and $p$ refer to electrons from the conduction and valence bands, respectively, and $E_{g}$ is the forbidden gap. We must find the matrix element,

$$
<\mathbf{k}_{1}\left|w^{+}\right| \mathbf{k}_{2}>=\frac{1}{(2 \pi)^{3}} \int d^{3} R \frac{e}{\varepsilon 2 i} e^{i \alpha} \hat{\mathbf{n}} \cdot \mathbf{D}_{0} \frac{1}{R^{2}} e^{-i\left(\mathbf{k}_{1}-\mathbf{k}_{2}\right) \cdot \mathbf{R}} .
$$

All integrals can be calculated analytically. After some algebra, one arrives at the expression,

$$
\begin{aligned}
& <\mathbf{k}_{1}\left|w^{+}\right| \mathbf{k}_{2}>=\frac{-1}{(2 \pi)^{3}} \frac{e e^{i \alpha}}{\varepsilon} D_{0} \cos \Theta(2 \pi) \int_{a}^{\infty} d R \frac{1}{q} \frac{d}{d R} \frac{\sin q R}{q R} \\
& =\frac{1}{(2 \pi)^{2}} \frac{e e^{i \alpha}}{\varepsilon} \frac{\mathbf{D}_{0} \cdot \mathbf{q}}{q^{2}} \frac{\sin q a}{q a} \stackrel{a \rightarrow 0}{\longrightarrow} \frac{1}{(2 \pi)^{2}} \frac{e e^{i \alpha}}{\varepsilon} \frac{\mathbf{D}_{0} \cdot \mathbf{q}}{q^{2}} .
\end{aligned}
$$

To estimate the absorption probability per time unit, the summation over all initial and final states in both bands must be performed. according the formula,

$$
\delta w=\int d^{3} k_{1} \int d^{3} k_{2}\left[f_{1}\left(1-f_{2}\right) w\left(\mathbf{k}_{1}, \mathbf{k}_{2}\right)-f_{2}\left(1-f_{1}\right) w\left(\mathbf{k}_{2}, \mathbf{k}_{1}\right)\right],
$$

where $f_{1}$ and $f_{2}$ represent the temperature-dependent distribution functions (Fermi-Dirac distribution functions) for the initial and final states, respectively. Emission and absorption are included, but for room temperature, one can assume that $f_{2} \simeq 0$ and $f_{1} \simeq 1$, leading to,

$$
\begin{aligned}
& \delta w=\frac{4}{3} \frac{\mu^{2}\left(m_{n}^{*}+m_{p}^{*}\right) 2\left(\hbar \omega-E_{g}\right) e^{2} D_{0}^{2}}{\sqrt{m_{n}^{*} m_{p}^{*} 2 \pi \hbar^{5} \varepsilon^{2}}} \int_{0}^{1} d x \frac{\sin ^{2}(x a \xi)}{(x a \xi)^{2}} \sqrt{1-x^{2}} \\
& =\frac{4}{3} \frac{\mu^{2}}{\sqrt{m_{n}^{*} m_{p}^{*}}} \frac{e^{2} D_{0}^{2}}{2 \pi \hbar^{3} \varepsilon^{2}} \xi^{2} \int_{0}^{1} d x \frac{\sin ^{2}(x a \xi)}{(x a \xi)^{2}} \sqrt{1-x^{2}} .
\end{aligned}
$$

In limiting cases, we finally obtain,

$$
\delta w=\left\{\begin{array}{l}
\frac{4}{3} \frac{\mu \sqrt{m_{n}^{*} m_{p}^{*}}\left(\hbar \omega-E_{g}\right) e^{2} D_{0}^{2}}{\text { f }}, \text { for } a \xi \ll 1, \\
\frac{4}{3} \frac{\mu^{3 / 2} \sqrt{2} \sqrt{\hbar \omega-E_{g}} e^{2} D_{0}^{2}}{a \hbar^{4} \varepsilon^{2}}, \text { for } a \xi \gg 1,
\end{array}\right.
$$

where the parameter $\xi=\frac{\sqrt{2\left(\hbar \omega-E_{g}\right)\left(m_{n}^{*}+m_{p}^{*}\right)}}{\hbar}$.

Note that the above formulae are distinct than the one for the ordinary photo-effect,

$$
\delta w_{0}=\frac{4 \sqrt{2}}{3} \frac{\mu^{5 / 2} e^{2}}{m_{p}^{* 2} \omega \varepsilon \hbar^{3}}\left(\frac{\varepsilon E_{0}^{2} V}{8 \pi \hbar \omega}\right)\left(\hbar \omega-E_{g}\right)^{3 / 2}
$$

Taking into account that the number of photons in the volume $V$ is equal to $\left(\frac{\varepsilon E_{0}^{2} V}{8 \pi \hbar \omega}\right)$, the probability of single-photon absorption by the semiconductor per time unit takes the following form in the ordinary photo-effect:

$$
q_{0}=\delta w_{0}\left(\frac{\varepsilon E_{0}^{2} V}{8 \pi \hbar \omega}\right)^{-1}=\frac{4(4) \sqrt{2}}{3} \frac{\mu^{5 / 2} e^{2}}{m_{p}^{* 2} \omega \varepsilon \hbar^{3}}\left(\hbar \omega-E_{g}\right)^{3 / 2},
$$

where the factor of 4 corresponds to the spin degeneracy of the band electrons.

The corresponding probability in the case of plasmon mediated transitions is given by Eq. (20). An enhancement of the transition probability for the near-field coupling with plasmons in comparison with direct photon absorption is related with admission of indirect interband transitions not restricted by momentum conservation, which does not hold in the nanostructure. This enhancement is gradually reducing with the radius $a$ increase, as expressed by Eq. (20).

Assuming now that dipole plasmon oscillations are excited by the rapid switching off the uniform electric field, $E(t)=$
$E_{0}(1-\Theta(t))$, the dipole-type solution for surface plasmons has the form [30],

$$
\mathbf{D}(t)=\left[0,0, D_{0} e^{-t / \tau^{\prime}} \cos \left(\omega_{1}^{\prime} t\right)\right]
$$

with

$$
D_{0}=\frac{e^{2} n_{e}}{m \omega_{1}^{2}} E_{0} \frac{4 \pi}{3} a^{3} .
$$

It is easy to estimate the total energy of this plasmon mode transfered to the semiconductor,

$$
\mathcal{A}=\beta \int_{0}^{\infty} \delta w \hbar \omega_{1} d t=\left\{\begin{array}{l}
\frac{2}{3} \frac{\beta \omega_{1} \tau^{\prime} \mu \sqrt{m_{n}^{*} m_{p}^{*}}\left(\hbar \omega_{1}-E_{g}\right) e^{2} D_{0}^{2}}{\hbar^{4} \varepsilon^{2}}, \text { for } a \xi \ll 1, \\
\frac{2}{3} \frac{\beta \omega_{1} \tau^{\prime} \mu^{3 / 2} \sqrt{2} \sqrt{\hbar \omega_{1}-E_{g}} e^{2} D_{0}^{2}}{a \hbar^{3} \varepsilon^{2}}, \text { for } a \xi \gg 1,
\end{array}\right.
$$

where $\beta$ accounts for the proximity constraints (not included in the model) that reduce the near-field contact of the sphere with the semiconductor; for the case of nanospheres deposited on the surface of a semiconductor layer, $\beta \sim \frac{h^{2}}{a^{2}} \sim 10^{-3}$ for $a \sim 50$ $\mathrm{nm}$ ( $h$ is the effective range of the near-field coupling), whereas for nanospheres entirely embedded in the semiconductor, $\beta$ is larger. Considering Eq. (24) and comparing the value given by the formula (25) with the energy loss estimated in [30] (the total energy of the plasmon oscillations), one finds that the damping rate of plasmons, $\tau^{\prime}$, induced by coupling with semiconductor,

$$
\frac{1}{\tau^{\prime} \omega_{1}}=\left\{\begin{array}{l}
\frac{4 \beta \mu \sqrt{m_{n}^{*} m_{p}^{*}}\left(\hbar \omega_{1}-E_{g}\right) e^{2} a^{3}}{3 \hbar^{4} \varepsilon}, \text { for } a \xi \ll 1, \\
\frac{4 \beta \mu^{3 / 2} \sqrt{2} \sqrt{\hbar \omega_{1}-E_{g}} e^{2} a^{2}}{3 \hbar^{3} \varepsilon}, \text { for } a \xi \gg 1 .
\end{array}\right.
$$

For nanospheres of $\mathrm{Au}$ deposited on an Si layer, we obtain,

$$
\frac{1}{\tau^{\prime} \omega_{1}}=44.092 \beta(a[n m])^{3} \frac{\mu}{m} \frac{\sqrt{m_{n}^{*} m_{p}^{*}}}{m},
$$

for $a \ll 0.15 \sqrt{m /\left(m_{n}^{*}+m_{p}^{*}\right)}[\mathrm{nm}]$, and

$$
\frac{1}{\tau^{\prime} \omega_{1}}=3.648 \beta(a[n m])^{2}\left(\frac{\mu}{m}\right)^{3 / 2},
$$

for $a \gg 0.15 \sqrt{m /\left(m_{n}^{*}+m_{p}^{*}\right)}[\mathrm{nm}]$, where light (heavy) carriers in $\mathrm{Si}, m_{n}^{*}=0.19(0.98) m$ and $m_{p}^{*}=0.16(0.52) m$, with $m$ being the bare electron mass; $\mu=\frac{m_{n}^{*} m_{p}^{*}}{m_{n}^{*}+m_{p}^{*}}$; and $E_{g}=1.14 \mathrm{eV}, \varepsilon=12$, and $\hbar \omega_{1}=2.72 \mathrm{eV}$. For these parameters and for nanospheres of radius $a$ in the range of $5-50 \mathrm{~nm}$, the case of Eq. (28) applies. The value of parameter $\beta$ taken from the experimental data is equal to approximately 0.002 .

One can also notice that for not sufficiently small values of $\beta$, the plasmon oscillator enters the overdamped regime due to extremely large efficiency of their coupling to band electrons. The energy transfer to the semiconductor substrate via the nearfield coupling channel is then so efficient that it quenches free plasmon oscillations but the energy flow to the semiconductor is maintained and can be estimated in the regime of the forced overdamped oscillator [36].

The efficiency of the plasmon near-field coupling channel may be displayed by the ratio of the probability of energy absorption in the semiconductor via the mediation of the surface plasmons (per single incident photon, $q_{m}$ ) to the probability of 
energy absorption in the semiconductor directly from the planewave illumination (also per single photon, $q_{0}$, as given by Eq. (22)). The probability $q_{m}$ can be expressed according to (20),

$$
q_{m}=\beta N_{m} \delta w\left(\frac{\varepsilon E_{0}^{2} V}{8 \pi \hbar \omega}\right)^{-1} .
$$

The driving force for plasmon oscillations is the electric field of the incident plane wave, whereas the damping force is the energy transfer described by $\frac{1}{\tau^{\prime}}$ as given above. The resulting red-shifted resonance, with a simultaneously reduced amplitude (as for damped and forced oscillator), corresponds to the stable balanced transfer of incident energy via plasmons to the substrate. Therefore,

$q_{m}=\left\{\begin{array}{l}\beta C_{0} \frac{128}{9} \pi^{2} a^{3} \frac{\mu \sqrt{\mu_{n}^{*} \mu_{p}^{*}}}{m^{2}}\left(\hbar \omega-E_{g}\right) \frac{e^{6} n_{e}^{2} \omega}{\hbar^{4} \varepsilon^{3}} f^{2}(\omega), \text { for } a \xi \ll 1, \\ \beta C_{0} \frac{128}{9} \sqrt{2} \pi^{2} a^{2} \frac{\mu^{3 / 2}}{m^{2}} \sqrt{\hbar \omega-E_{g}} \frac{e^{6} n_{e}^{2} \omega}{\hbar^{3} \varepsilon^{3}} f^{2}(\omega), \text { for } a \xi \gg 1,\end{array}\right.$

where $f(\omega)=\frac{1}{\sqrt{\left(\omega_{1}^{2}-\omega^{2}\right)^{2}+4 \omega^{2} / \tau^{\prime 2}}}$ corresponds to the amplitude factor for the forced and damped oscillator and $D_{0}=$ $\frac{e^{2} n_{e} E_{0} 4 \pi a^{3}}{3 m} f(\omega) ; C_{0}=\frac{N_{m} 4 / 3 \pi a^{3}}{V}, V$ is the volume of the semiconductor, $N_{m}$ is the number of metallic nanospheres, and $\xi=\frac{\sqrt{2\left(\hbar \omega-E_{g}\right)\left(m_{n}^{*}+m_{p}^{*}\right)}}{\hbar}$.

The ratio $\frac{q_{m}}{q_{0}}$ can be thus written as,

$$
\frac{q_{m}}{q_{0}}=\left\{\begin{array}{l}
\frac{8 \pi^{2} a^{3} \beta C_{0} \sqrt{m_{n}^{*}}\left(m_{p}^{*}\right)^{5 / 2} e^{4} n_{e}^{2} \omega^{2} f^{2}(\omega)}{3 \sqrt{2} \mu^{3 / 2} m^{2} \sqrt{\hbar \omega-E_{g}} \hbar^{3} \varepsilon^{2}}, \text { for } a \xi \ll 1, \\
\frac{8 \pi^{2} a^{2} \beta C_{0}\left(m_{p}^{*}\right)^{2} e^{4} n_{e}^{2} \omega^{2} f^{2}(\omega)}{3 \mu m^{2}\left(\hbar \omega-E_{g}\right) \hbar^{2} \varepsilon^{2}}, \text { for } a \xi \gg 1 .
\end{array}\right.
$$

The above results are consistent with the experimental behavior $[3,30,36]$. To compare with the experiment measurements we estimate the photocurrent in the case of a silicon metallically modified photodiode $I^{\prime}=|e| N\left(q_{0}+q_{m}\right) A$, where $N$ is the number of incident photons; $q_{0}$ and $q_{m}$ are the probabilities of single-photon absorption in the ordinary photo-effect and of single-photon absorption mediated by the presence of metallic components, respectively; and $A$ is the amplification factor [47]. The normalized photo-current (measured in the experiment [3]) can be thus expressed as follows,

$$
\frac{I^{\prime}}{I}=1+\frac{q_{m}}{q_{0}}
$$

where $I=I^{\prime}\left(q_{m}=0\right)$ and the ratio $q_{m} / q_{0}$ is given by Eq. (31).

It is worth noting that the strong photocurrent enhancement was observed at the relatively low surface concentration of metallic nanocomponents. However, in many real solar cells, experimentally observed enhancements due to metallic nanocomponents are not so pronounced [7]. This is because some additional effects contribute in a competitive manner, such as the reflection of incident photons or destructive collective interference in a dense metallic coverage $[4,15]$, surface state perturbations caused by deposited nanoparticles, specific type of deposition and other. These effects are phenomenologically accounted for by the fitted experimental factor $\beta$.

\section{NUMERICAL MODELING UPON IMPROVED COMSOL}

\section{A. Modification of the dielectric function of nanoparticles with plasmon}

The wide spread off in the literature numerical calculations of absorption enhancement due to incorporation of metallic nanoparticles in the solar cell are performed using as a prerequisite the material optical parameters taken from various experimental data. They are, however, addressed to bulk materials or rarely for thin films and do not display important behavior genuine for nanoparticles. Their behavior can be significantly different since in the nano-scale some quantum effects becomes to be especially important in described above.

The dielectric function can be divided into real and imaginary part $\varepsilon=\varepsilon^{\prime}+\varepsilon^{\prime \prime}$ which are connected to refractive index and absorption coefficient, respectively.

$$
\varepsilon^{\prime \prime}=\frac{n c}{\omega} \alpha(\omega)
$$

where $n$ is a real part of the refractive index and $\alpha$ is the absorption coefficient of the semiconductor defined as

$$
\alpha(\omega)=\frac{\hbar \omega q_{m(0)}}{I_{0}}
$$

where and $I_{0}$ is the incident electromagnetic energy flux and $q_{m(0)}$ is the number of transitions per unit volume and time defined according to eq. 29 for the case of Si with metallic nanoparticles on the top and eq. 22 in the case without nanoparticles. The photon absorption probability was obtained using Fermi Golden Rule as described in the previous paragraphs of the present paper. For nanoparticles of size $5-55 \mathrm{~nm}$ and $\mathrm{Si}$ substrate the parameter $a \xi$ is bigger than 1 , so we use the second case of eq. 20.

The plasmon amplitude $D_{0}$ can be obtained from the formula for total power of a dipole radiation,

$$
D_{0}^{2}=\frac{4 \pi \varepsilon_{0} \lambda^{4}}{(2 \pi)^{4} \mathcal{C}} \int_{\Sigma} \mathbf{S} \cdot \mathbf{d} \sigma
$$

where $\mathbf{S}$ Poynting vector and $\Sigma$ is the nanoparticle surface. The total energy dissipation inside the dispersive material can be calculated as [48]:

$$
Q=\frac{\omega}{4 \pi}\left(\varepsilon^{\prime \prime} \mathbf{E}^{2}+\mu^{\prime \prime} \mathbf{H}^{2}\right) .
$$

The absorption enhancement $\mathcal{A}$, may be defined as the ratio of absorption with and without nanoparticles and is calculated according to eq.36 by integrating of square of the normal electric field component over the semiconductor substrate volume:

$$
\mathcal{A}(\omega)=\frac{\frac{\omega}{4 \pi} \int_{V} n \varepsilon_{m}^{\prime \prime}(\omega) \mathbf{E}_{\text {with NP }}^{2} d V}{\frac{\omega}{4 \pi} \int_{V} n \varepsilon_{0}^{\prime \prime}(\omega) \mathbf{E}_{\text {without NP }}^{2} d V}
$$

where $\varepsilon_{m(0)}^{\prime \prime}$ is the imaginary part of dielectric function modified for the case with and without nanoparticles, respectively. In the calculations based on the measured refractive index of $\mathrm{Si}$ (after [49] ) the imaginary part of dielectric function of Si was defined as $\varepsilon^{\prime \prime}(\omega)=2 n(\omega) k(\omega)$.

\section{B. Calculation model setup}

In our calculations we have used the finite element method (FEM) implemented in the commercial software COMSOL Multiphysics 5.0, specifically we have worked with Wave Optics module (http:/ / www.comsol.com).

The simulation cell consisted of three domains: air at the top, semiconductor at the bottom and metallic nanoparticle at the center laying on the semiconductor substrate.

The calculations were conducted in two steps: first within full field formulation we have calculated the distribution for the electromagnetic field without the nanoparticle and then, in the 
second step, we have used this field as the background field in the scattering field formulation for the structure with the nanoparticle.

In the first step, within the full field formulation the incident light was set as a planar TM wave propagating along z-axis. To do so, we have used two ports: the first for defining incoming plane wave parameters (on the top boundary) and the second to absorb wave transmitted through the substrate (at the bottom). On the side boundaries we defined the Floquet boundary conditions, which allowed us to effectively simulate an infinite system.

In the second step instead of periodic conditions, we have defined additional domain surrounding the calculation cell and absorbing all outgoing light (so called Perfectly Matched Layers). Such an approach allowed us to reduce the simulation area and to effectively simulate rare nanoparticle coverings, neglecting, however, interparticle interaction. This is justified only for sufficiently diluted coverings, just as in the experiment [3], where planar density of metallic nanoparticles is of order $10^{7}-10^{8} / \mathrm{cm}^{2}$. For so distant separation of nanoparticles there are unimportant also corrections induced by a regular or random distribution of metallic compounds. We have verified [50] that the collective effects including destructive interference of incident light may affect the plasmon photovoltaic effect at much larger (by several orders) densities of coverages, so these effects can be safely neglected in the presented here simulation. The thickness of the Si substrate and the width of the computational cell were set $400 \mathrm{~nm}$, where the thickness of the air domain was assumed 300 $\mathrm{nm}$. The mesh size was adjusted to the refractive index of the air and $\mathrm{Si}$ domains in such a way to ensure at least 5 elements per wavelength. The nanoparticle was mashed with element size $a / 5$.

The optical properties of the nanoparticle and substrate was modeled by the dielectric function. We have performed calculations with dielectric function of Si modified on the basic of FGR as described in previous sections of this article and compared them with calculations performed using the measured dielectric data of Si from [49]. The dielectric function of the Au was modeled by Drude model with parameters $\varepsilon_{\infty}=9, \omega_{p}=9 \mathrm{eV}$ and damping $\gamma$ defined as in eq.28.

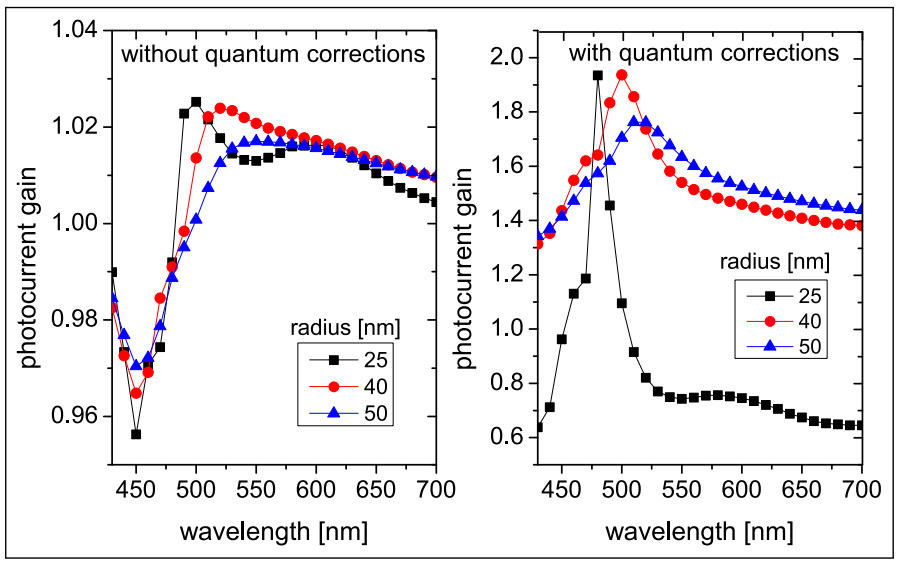

Fig. 2. Photo-current gain calculated without (left panel) and with (right panel) quantum-corrected Comsol simulations for Au nanoparticles radii 25, 40 and $50 \mathrm{~nm}$ with planar concentrations $6.6 \times 10^{8}, 1.6 \times 10^{8}$ and $0.77 \times 10^{8} 1 / \mathrm{cm}^{2}$, respectively (as in experiment [3] — cf. Fig.3).

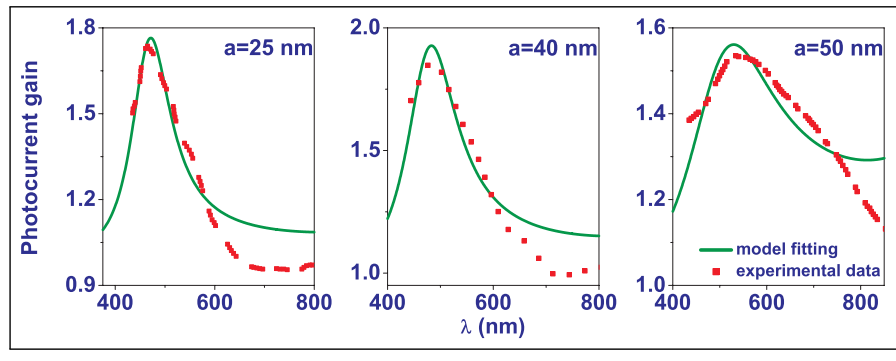

Fig. 3. Dependence of the normalized photocurrent $\frac{I^{\prime}}{I}(\lambda)$ given by the analytical formulae (32) and (31): comparison with the experimental data (red) after [3] for (left) $a=25 \mathrm{~nm}$ and $n_{s}=6.6 \times 10^{8} 1 / \mathrm{cm}^{2}$, (center) $a=40 \mathrm{~nm}$ and $n_{s}=$ $1.6 \times 10^{8} 1 / \mathrm{cm}^{2}$, and (right) $a=50 \mathrm{~nm}$ and $n_{s}=0.8 \times 10^{8}$ $1 / \mathrm{cm}^{2}(H=3 \mu \mathrm{m})$.

\section{Exemplary results and comparison with experiment}

In order to demonstrate feasibility and accuracy of the improved Comsol simulation we present our simulation of the experimental setup as in [3] including into Comsol calculus the described above formulae for plasmon damping due to coupling of plasmons with band electrons in the semiconductor substrate. The quantumly improved Comsol simulation-Fig. 2-very well reproduces the experimental data (after [3]) with respect to proper size dependence of the photo-current gain ( $\frac{I}{I^{\prime}}$ plotted in Fig. 2) in satisfactory coincidence with the experiment for the maxima positions, heights and widths of spectra for different radii of metallic nanoparticles.

The pretty good coincidence with the same experimental data is also achieved by application of our analytical approach given by Eqs. (32) and (31), as visualized in Fig. 3. The apparent agreement of the numerical simulation, analytical theory and of the experimental data proves the consistency and significance of the presented quantum approach to modeling of plasmon photo-voltaic effect. Noticeable is the success of here formulated quantum upgrade of the Comsol software, which considerably increases its usability, fidelity and precision in the field of nanoplasmonics for photovoltaics.

\section{INCLUSION OF NON-LOCAL OPTICAL RESPONSE IN METALLIC NANOSPHERES}

Besides of quantum effects in plasmon mediation of the photovoltaic effect in metallically modified solar cells, we also address to another quantum type problem arising in nonlocal plasmon response in metallic nanoparticles. We examine this effect governed by the electron microscopic dynamics in metallic nanoparticles in order to assess a nanoparticle size at which the nonlocal effect is important and to evaluate a potential role of nonlocal quantum corrections in plasmon aided photovoltaic effect.

The nonlocal effects are spatially governed by the dispersion of plasmons and by particularities of electron dynamics on the scale of a metallic nanoparticle, se we denominate effects of electron coupling over a short distance [51]. The details of microscopic short distance electron interactions are inherent to the solution for the displacement field $\vec{D}$ of the Coulomb equation,

$$
\begin{aligned}
\nabla \vec{D}(t, \vec{r}) & =\epsilon(\vec{r}) \vec{E}(t, \vec{r}) \\
\Rightarrow \vec{D}(t, \vec{r}) & =\int d r^{\prime} \epsilon\left(\vec{r}, \vec{r}^{\prime}\right) \vec{E}\left(t, r^{\prime}\right) .
\end{aligned}
$$


In homogeneous media, we can assume a dependence on the distance $\left|\vec{r}-\vec{r}^{\prime}\right|$ rather than on the specific position of electrons, which allows to solve Maxwell's equations in Fourier space

$$
\vec{D}(\omega, \vec{k})=\epsilon(\omega, \vec{k}) \vec{E}(\omega, \vec{k})
$$

The dependence on the wave vector $\vec{k}$ enables us to describe nonlocal electron-electron interaction (Coulombic force) and electron diffusion effects. It is important to note that the large$k$ response that originates in the sub-wavelength oscillations of plasmonic excitations is not only an inherent prerequisite for many intriguing wave phenomena, but also particularly sensitive to nonlocality. However, the common local response approximation of material parameters has no upper wavelength cut-off, does suppress short-range electron interactions which can strongly dampen the response beyond $\omega / v_{F}$.

Advances in fabrication of nanostructures and experimental access to particle sizes and interparticle spacings below $10 \mathrm{~nm}$ to directly or indirectly observe such effects [52-59] have led to an increased interest in semi-classical approaches towards the incorporation of damping and interaction effects stemming from the quantum nature of charge carriers. Efforts to extend the original formulation of light scattering by a sphere by Gustav Mie [19] which excludes electro-optical bulk and surface effects of the conduction band electrons are made since the 1980s [6064]. Advanced material models can be derived from perturbative theories $[23,51]$, by separating the free electron dynamics from the core electron polarization via the hydrodynamic equation for an electron plasma [22-26, 65-73] and from microscopic theories [30, 36, 74]. It was shown within the hydrodynamic framework that the electron spill-out can be adequately incorporated [75].

We show in the corresponding section below that accounting for nonlocal response obtained with the hydrodynamic approach leads to longitudinal pressure waves as additional solutions to the combined system of differential equations of the electromagnetic wave equation and (linearized) Navier-Stokes equation. These waves primarily create additional damping channels, however, they also yield resonant enhancement effects [26, 71].

\section{A. Electron dynamics with the hydrodynamic model}

In recent years, a great effort to theoretically [22-26, 65-73] describe and subsequently to experimentally $[55-57,59]$ verify the effect of spatial dispersion in metals was made. In the hydrodynamic approach, coupling the electromagnetic wave equation

$$
\nabla \times \nabla \times \vec{E}-k^{2} \varepsilon_{b} \vec{E}=\frac{4 \pi i k^{2}}{\omega} j^{\text {ind }}
$$

to the (linearized) Navier-Stokes equation

$$
\vec{j}^{\text {ind }}=\frac{i}{\omega+i \gamma_{p}}\left(\frac{\omega_{p}^{2}}{4 \pi} \vec{E}-\left(\beta^{2}+D\left(\gamma_{p}-i \omega\right)\right) \nabla \rho^{\text {ind }}\right)
$$

allows treating the conduction band electrons as a plasma subject to short-ranged interaction such as the Coulomb force included in the pressure term $p=\beta^{2} \rho^{\text {ind }}$ and electron diffusion via the diffusion coefficient $D$. It is convenient to abbreviate $\beta_{\text {GNOR }}^{2}=\beta^{2}+D\left(\gamma_{p}-i \omega\right)$ (where GNOR refers to the generalized nonlocal optical response model $[24,25])$. With this, we can write the wave equation in a compact form

$$
\nabla^{2} \vec{E}+k^{2} \varepsilon_{\perp} \vec{E}=\eta \rho^{\text {ind }}
$$

where $\eta \equiv 4 \pi\left(\frac{1}{\epsilon_{b}}-\frac{k^{2} \beta_{\mathrm{GNOR}}^{2}}{\omega(\omega+i \gamma)}\right)$ and $\epsilon_{\perp}=\epsilon_{b}-\frac{\omega_{p}^{2}}{\omega\left(\omega+i \gamma_{p}\right)}$. Together with the continuity equation $\nabla \vec{j}^{\text {ind }}=i \omega \rho^{\text {ind }}$, we readily obtain a separate wave equation for the induced charges

$$
-\beta_{\mathrm{GNOR}}^{2} \nabla^{2} \rho^{\text {ind }}=\frac{\epsilon_{\perp}}{\epsilon_{b}} \omega\left(\omega+i \gamma_{p}\right) \rho^{\text {ind }},
$$

where $\nabla \vec{E}=\frac{4 \pi}{\epsilon_{b}} \rho^{\text {ind }}$ was used. This yields the wave vector of the longitudinal field and motion of electrons

$$
q=\frac{1}{\beta_{\mathrm{GNOR}}} \sqrt{\frac{\epsilon_{\perp}}{\epsilon_{b}} \omega\left(\omega+i \gamma_{p}\right)} .
$$

Thus, this system of coupled equations yields an additional wave solution, longitudinal in character, and can be solved for different geometries leading to nonlocal extensions of e. g., Mie [22, 23] and Fresnel coefficients [71]. Typically, hard-wall boundary conditions are assumed for the additional boundary condition $\vec{j}^{\text {ind }} \equiv 0$ prohibiting electrons to trespass into the dielectric surrounding, using a uniform electron density $n_{0}$ inside the material and neglecting the electron spill-out. However, it was shown that a smooth surface distribution of electrons can be taken into account accurately [75] and that the hydrodynamic model is capable of dealing with the spill-out by solving the above equations with position-dependent material parameters $\omega_{p}(z)^{2}=4 \pi n_{0}(z) \frac{e^{2}}{m_{e}}$. Note that the finite value of $\Im(1 / q)$ leads to a finite distribution of induced boundary charges upon illumination in contrast to the delta-like accumulation at the surface within classical theory. The characteristic penetration depth $\Im(1 / q)$ is hereby comparable to the electron spill-out $[23,76]$.

Next, we present the derivation of nonlocal Mie scattering coefficients of individual spheres and nanoshells described with the hydrodynamic model [23]. The resulting scattering matrices can be used to investigate interacting spheres with a multiple scattering method [77]. The hydrodynamic model has no free parameters which makes the resultant nonlocal response for the short distances involved in the interaction between the charges of metallic nanoparticles the sole source of these effects, in contrast to the quantum-confinement picture for plasmon broadening presented by of Kreibig.

The derivation of the nonlocal Mie coefficients for spherical geometries starts from Eq. (42) which describes the evolution of the electric field, together with Eq. (43) which is the wave equation for the induced charge.

It is convenient to use an expansion of the electric field into scalar functions [78] as

$$
\vec{E}=(1 / k) \nabla \psi^{L}+\vec{L} \psi^{M}+\frac{\nabla \times \vec{L}}{k i} \psi^{E},
$$

where $\vec{L}=-i \hbar \vec{r} \times \nabla$ is the angular momentum operator, and the superscripts $E, M$, and $L$ indicate electric, magnetic, and longitudinal components, respectively. The additional boundary condition, Eq. (41), becomes with $\hat{\vec{r}} \vec{j}=0$

$$
\beta^{2} \frac{\partial}{\partial r} \rho^{\text {ind }}=\frac{e^{2} n_{0}}{m_{e} k}\left(\frac{\partial}{\partial r} \psi^{L}+\frac{1}{r} l(l+1) \psi^{E}\right)
$$

in terms of the scalar functions and the angular momentum number $l$ using the identity $-\vec{r} \cdot(\nabla \times \vec{L})=(-i \vec{r} \times \nabla) \cdot \vec{L}=L^{2}=$ $l(l+1)$. The boundary conditions for the electric and magnetic field components result in the continuity of $\psi^{M},\left(1+r \frac{\partial}{\partial r}\right) \psi^{M}$, $\psi^{L}+\left(1+r \frac{\partial}{\partial r}\right) \psi^{E}$, and $\epsilon \psi^{E}$ for the scalar functions. 


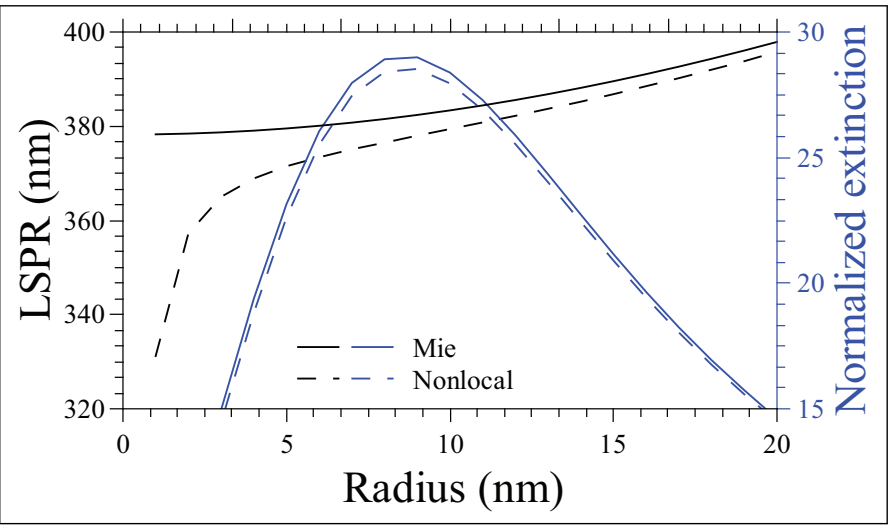

Fig. 4. Effect of spatial dispersion on gold nanoparticles: A strong blueshift of the localized surface plasmon resonance (LSPR, black) is observed for small metallic nanoparticles with respect to the common local response approximation (Mie, solid). Furthermore, the inherent damping effects in nonlocal response theory (dashed) lead to a reduction of the optical response as shown here for the extinction cross section (blue) normalized to the illuminated surface area.

The magnetic and electric scalar functions $\psi^{v}(v=\{E, M\})$ obey a Helmholtz equation of the form $\left(\nabla^{2}+k^{2} \epsilon_{\perp}\right) \psi^{v}=0$ and can therefore be expanded in terms of spherical Bessel functions

$$
\psi^{v}=\sum_{L} \psi_{L}^{v} j_{L}\left(k_{\perp} r\right)
$$

Similarly, the electron density is expanded into

$$
\rho^{\text {ind }}(\vec{r}, \omega)=\sum_{L} \rho_{L} j_{L}(q r),
$$

with the longitudinal wave vector $q$ given by Eq. (44). The longitudinal scalar function satisfies a different wave equation, namely $\nabla^{2} \psi^{L}=4 \pi k / \epsilon_{b}$, which we find from the Coulomb law $\nabla \epsilon_{b} \vec{E}=4 \pi \rho^{\text {ind }}$. Note that the above analysis is needed for the metal region, where the electric $(v=E)$ and magnetic $(v=M)$ field are given by $A_{l}^{v} j_{L}$, with $j_{L}=j_{l m}\left(k_{\perp} r\right)$. Outside the particle, the longitudinal scalar function vanishes since there are no induced charges in the dielectric surrounding. Therefore, the electric scalar field is given by $j_{l m}\left(k_{0} r\right)+t_{l}^{v} h_{l m}^{+}\left(k_{0} r\right)$ with unknown parameters $A_{l}^{v}$ and scattering matrix $t_{l}^{v}$. Exploiting the boundary conditions stated above, we find a set of linear equations for the magnetic and electric scattering matrices. Interestingly, the magnetic scattering matrix is unchanged with respect to the local theory, indicating that magnetic modes are not sensitive to the induced longitudinal modes. The scattering matrix for the electric scalar function is more complicated than in the local approximation due to the appearance of $\psi^{L}$ in the metal region that contains information on the nonlocal response. The additional boundary condition yields a prescription to calculate $\rho_{L}$.

The local scattering matrix can then be extended by a single parameter describing nonlocal behavior of the electron motion in the conduction band

$$
g_{l}=\frac{l(l+1) j_{l}\left(\theta_{\perp}\right) j_{l}(q a)}{q a j_{l}^{\prime}(q a)}\left(\frac{\epsilon_{\perp}}{\epsilon_{b}}-1\right)
$$

and becomes with $\theta_{0}=k a \sqrt{\epsilon_{0}}$ and $\theta_{\perp}=k a \sqrt{\epsilon_{\perp}}$.

$$
t_{l}^{E}=\frac{-\epsilon_{\perp} j_{l}\left(\theta_{\perp}\right)\left[\theta_{0} j_{l}\left(\theta_{0}\right)\right]^{\prime}+\epsilon_{0} j_{l}\left(\theta_{0}\right)\left(\left[\theta_{\perp} j_{l}\left(\theta_{\perp}\right)\right]^{\prime}+g_{l}\right)}{\epsilon_{\perp} j_{l}\left(\theta_{\perp}\right)\left[\theta_{0} h_{l}^{+}\left(\theta_{0}\right)\right]^{\prime}-\epsilon_{0} h_{l}^{+}\left(\theta_{0}\right)\left(\left[\theta_{\perp} j_{l}\left(\theta_{\perp}\right)\right]^{\prime}+g_{l}\right)},
$$

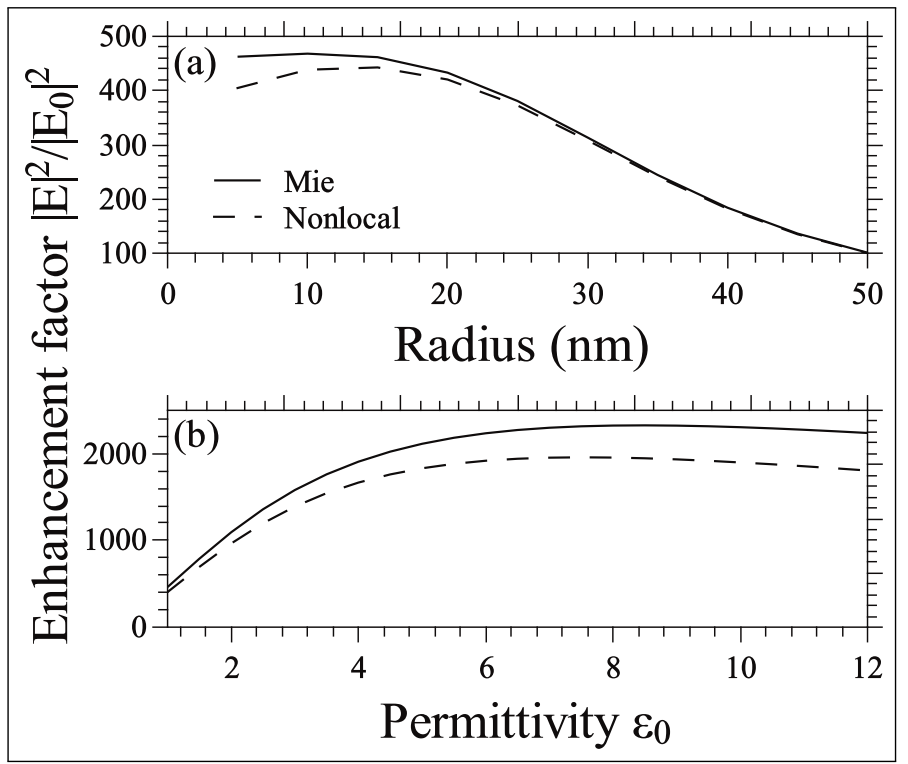

Fig. 5. Reduction of the maximum field enhancement of gold nanoparticles with spatial dispersion: Maximum field enhancement factor $E F=|\vec{E}|^{2} /\left|\vec{E}_{0}\right|^{2}$ as a function of (a) particle radius and (b) permittivity of the surrounding medium.

where the primes indicate differentiation with respect to the $\theta$ variables. The scattering coefficients $t_{l}^{v}$ fully contain the optical response of the particle for an external observer.

Note that the nonlocal parameter $g$ vanishes under the assumption of local response $\left(\beta \rightarrow 0 \Rightarrow g_{l} \rightarrow 0\right)$ fully recovering the original Mie coefficients $[19,79]$. This allows us to study nanoparticles with only a small correction in available numerical procedures.

Likewise, for a nonlocal metal nanoshell the magnetic response is insensitive to the nonlocal properties of the material. The electric part, however, mixes with the longitudinal components from the two interfaces of the metal intermediate layer. For the electric scalar functions, we obtain a linear system of six equations that can be solved with linear algebra in practice.

The expressions obtained are used to calculate e. g. the extinction cross section of an individual sphere via

$$
\sigma_{\mathrm{ext}}=\frac{2 \pi}{k^{2} \epsilon_{0}} \sum_{l}(2 l+1) \operatorname{Im}\left(\mathrm{t}_{1}^{\mathrm{E}}+\mathrm{t}_{1}^{\mathrm{M}}\right) .
$$

\section{B. Comments on the significance of nonlocal plasmon effects}

The main observation for nonlocal theories are a blueshift of the plasmon resonance with respect to the common local approximation, see Fig. 4, and plasmon broadening, in particular tied to the diffusion coefficient which can be set to fully capture the broadening found with Kreibig damping [24, 25]. In the present work, we have $D \equiv 0$. The damping associated with nonlocal response is shown in Fig. 5 in terms of the local field enhancement.

The presented semi-classical approach towards inclusion of aspects of mesoscale electron dynamics in metal nanoparticles has the advantage of analytic expressions fully compatible with existing computational procedures. The scattering matrix presented here can be used in standard Mie calculations and also to obtain optical properties of complex structures, e. g. with a multiple scattering approach [77].

The presented nonlocal theory introduces a novel type of 
electron motion, longitudinal pressure waves, in addition to the transversal modes stemming from the classical electromagnetic wave equation. This additional electronic excitation offers further damping channels due to energy lost in this dampened motion. Here, the Mie coefficients were derived from the coupled system of optical and electronical excitation yielding modified scattering matrices that can again be implemented in existing methods. The properties of the longitudinal wave are given by analytic expressions such as their wave vector (Eq. (44)) and their importance with respect to the common Mie solution is entirely captured in the additional term (Eq. (47)).

It is noteworthy that the hydrodynamic theory allows fully retarded calculations for not only spherical particles as reviewed here, but also planar geometries (nonlocal Fresnel coefficients) [71] and even regular two-dimensional particle layers [26]. Although the presented quantum phenomena are highly localized they can have a strong impact on a larger particle or system in the interplay with long-range retardation effects. In addition, particle layer modes can couple to nonlocal modes within particle arrays and thus increase their impact on a larger scale [26].

Nevertheless, from the above presented analysis it follows that nonlocal quantum corrections do not change significantly the Mie-response for metallic nanoparticles of size as are utilized for metallically modified solar cells. The strongest nonlocal effect is a large blue-shift of plasmon resonance in nanoparticles below $3 \mathrm{~nm}$ for their radius (cf. Fig. 4 and Fig. 5). Despite this shift in frequency the related correction to plasmon attenuation is rather small and far lower than the irradiation induced plasmon damping discussed in paragraphs $2-3$ of the present paper. So we can conclude that nonlocal quantum corrections to plasmon response do not modify significantly the plasmon aided photovoltaic effect, though nonlocal effects elucidate some previously unclear spectral property of ultra-small nanoparticles (up to ca. $3 \mathrm{~nm}$ for radius) yielding the blue-shift of the resonance, opposite to the red-shift caused by the ordinary spill-out in the same scale of nanoparticle size. [29].

\section{SUMMARY}

Quantum effects due to dipole near-field coupling of surface plasmons in metallic nanoparticles with band electrons in the semiconductor substrate have been accounted for in terms of the Fermi golden rule in order to determine damping rate of plasmons in the regime of plasmon aided ptoto-effect. This coupling opens a very efficient short-time channel for energy transfer from plasmons to electrons which results in giant damping of plasmons. The effect strongly depends on the size of nanoparticles deposited on the top of the solar cell. The trade-off in efficiency growth with respect to opposite size effects is demonstrated and optimal metallic component size is identified for photovoltaic plasmon applications. The found analytical formula for the damping rate is utilized to upgrade the Drude-Lorentz formula for the dielectric function of the metallic component. The improved dielectric function has been next input as a prerequisite to numerical simulation of solar cell metallically modified upon the commercial software-system Comsol. The strong corrections are evidenced in comparison to previous conventional Comsol approach neglecting damping of plasmons due to their quantum coupling in the near field-zone with electrons. The results evidenced high significance of the presented quantum improvement of former classical wide spread off numerical tools like Comsol or Mie-type packets.
We have also looked at different types of damping mechanisms, such as electron collision, irradiation and interaction effects. We found analytic expressions compatible with existing numerical procedures. For the smallest nanoparticles with radii of few $\mathrm{nm}$, not exceeding $10 \mathrm{~nm}$, the spatial dependence of electron scattering on other electrons, phonons, defects and admixtures and on boundary of the metallic nanoparticle characterizes the dissipation of energy of plasmons and yields a damping induced correction in the spectral position of the surface plasmon frequency in agreement with experiments. For larger particles (with radii beyond $10 \mathrm{~nm}$ ), the plasmon damping rapidly grows and is overwhelming by the Lorentz friction of plasmon oscillations. The related irradiation energy losses dominate over other channels of plasmon damping in nanoparticles with radii larger than $10 \mathrm{~nm}$ in the case of free plasmon oscillations (when the nanoparticle is placed in vacuum or immersed in a dielectric medium). The irradiation losses additionally strengthen when in the vicinity of the metallic nanoparticle another electrically active system is present, e.g., a band electron system in a substrate semiconductor on which the metallic nanoparticle can be deposited. Near-field coupling of plasmons with the substrate band electrons opens a very effective energy transfer channel resulting in a giant damping of plasmons for all size-scale of metallic nanoparticles, though with specific size dependence identified by the Fermi golden rule. This damping is greater than plasmon attenuation caused by the Lorentz friction of plasmons in a dielectric medium or vacuum and allows for plasmon mediation in the photovoltaic effect. Inclusion of this size dependent damping in the dielectric function for a metallic nanoparticle used in the Mie approach to plasmons or in the other version of Maxwell equation solution (typically by finite element method for solution of differential equations as applied in the commercial system Comsol) appears to be crucial for any reliable utilization of these methods to model plasmon effect in metallically improved solar cells. Though details of the e-m field distribution are precisely displayed by the Comsol software which is of high importance in the case of various size, arbitrary shape and deposition type of metallic nanoparticles, the negligence of the imaginary part of the plasmon energy (its damping) caused by described above irradiation effects leads to a very big error. Thus, for a reliable fit of numerical simulation (of Comsol or Mie type) with experimental observations of plasmon aided solar cells, the inclusion of the irradiation induced plasmon damping, highly exceeding the electron scattering rate, is necessary.

Supported by COST Action MP1406, DFG Research Fellowship (grant no. 268910011) by the German Research Foundation (Deutsche Forschungsgemeinschaft DFG) and Polish Ministry of Science (MNiSW grant no. 0731/E-366/STYP/1/11/2016/2017).

\section{REFERENCES}

1. K. Okamoto, I. Niki, A. Scherer, Y. Narukawa, and Y. Kawakami, "Surface plasmon enhanced spontaneous emission rate of InGaN/ GaN quantum wells probed by time-resolved photoluminescence spectroscopy," Appl. Phys. Lett. 87, 071102 (2005).

2. S. Pillai, K. R. Catchpole, T. Trupke, G. Zhang, J. Zhao, and M. A. Green, "Enhanced emission from Si-based light-emitting diodes using surface plasmons," Appl. Phys. Lett. 88, 161102 (2006).

3. D. M. Schaadt, B. Feng, and E. T. Yu, "Enhanced semiconductor optical absorption via surface plasmon excitation in metal nanoparticles," Appl. Phys. Lett. 86, 063106 (2005).

4. S. P. Sundararajan, N. K. Grandy, N. Mirin, and N. J. Halas, "Nanoparti- 
cle enhancement and suppression of photocurrent in a silicon photodiode," Nano Lett. 8, 624 (2008).

5. M. Westphalen, U. Kreibig, J. Rostalski, H. Lüth, and D. Meissner, "Metal cluster enhanced organic solar cells," Sol. Energy Mater. Sol. Cells 61, 97 (2000).

6. A. J. Morfa, K. L. Rowlen, T. H. Reilly, M. J. Romero, and J. Lagemaat, "Plasmon-enhanced solar energy conversion in organic bulk heterojunction photovoltaics," Appl. Phys. Lett. 92, 013504 (2008).

7. M. A. Green and S. Pillai, "Harnessing plasmonics for solar cells," Nat. Photon. 6, 130-132 (2012).

8. L. Hong, Rusli, X. Wang, H. Zheng, L. He, X. Xu, H. Wang, and H. Yu, "Design principles for plasmonic thin film GaAs solar cells with high absorption enhancement," J. of Appl. Phys. 112, 054326 (2012).

9. M. Jeng, Z. Chen, Y. Xiao, L. Chang, J. Ao, Y. Sun, E. Popko, W. Jacak, and L. Chow, "Improving efficiency of multicrystalline silicon and CIGS solar cells by incorporating metal nanoparticles," Materials 8, 6761 (2015).

10. H. A. Atwater and A. Polman, "Plasmonics for improved photovoltaic devices," Nat. Materials 9, 205 (2010).

11. D. Derkacs, S. H. Lim, P. Matheu, W. Mar, and E. T. Yu, "Improved performance of amorphous silicon solar cells via scattering from surface plasmon polaritons in nearby metallic nanoparticles," Appl. Phys. Lett. 89, 093103 (2006).

12. S. Kim, S. Na, J. Jo, D. Kim, and Y. Nah, "Plasmon enhanced performance of organic solar cells using electrodeposited ag nanoparticles," Appl. Phys. Lett. 93, 073307 (2008).

13. M. Kirkengen, J. Bergli, and Y. M. Galperin, "Direct generation of charge carriers in C-Si solar cells due to embedded nanoparticles," J. of Appl. Phys. 102, 093713 (2007).

14. J. Lee and P. Peumans, "The origin of enhanced optical absorption in solar cells with metal nanoparticles embedded in the active layer," Opt. Express 18, 10078 (2010).

15. M. Losurdo, M. M. Giangregorio, G. V. Bianco, A. Sacchetti, P. Capezzuto, and G. Bruno, "Enhanced absorption in Au nanoparticles/a-Si:H/cSi heterojunction solar cells exploiting au surface plasmon resonance," Sol. Energy Mater. Sol. Cells 93, 1749 (2009).

16. K. R. Catchpole, S. Mokkapati, F. Beck, E. Wang, A. McKinley, A. Basch, and J. Lee, "Plasmonics and nanophotonics for photovoltaics," MRS Bulletin 36, 461-467 (2011).

17. J. Wu, S. C. Mangham, V. Reddy, M. Manasreh, and B. Weaver, "Surface plasmon enhanced intermediate band based quantum dots solar cell," Solar Energy Materials and Solar Cells 102, 44-49 (2012).

18. N. Kalfagiannis, P. Karagiannidis, C. Pitsalidis, N. Panagiotopoulos, C. Gravalidis, S. Kassavetis, P. Patsalas, and S. Logothetidis, "Plasmonic silver nanoparticles for improved organic solar cells," Solar Energy Materials and Solar Cells 104, 165-174 (2012).

19. G. Mie, "Beitrige zur Optik trüber Medien, speziell kolloidaler Metallösungen," Ann. Phys. 25, 376 (1908).

20. C. F. Bohren and D. R. Huffman, Absorption and Scattering of Light by Small Particles (Wiley, New York, 1983)

21. D. Pines, Elementary Excitations in Solids (ABP Perseus Books, Massachusetts, 1999)

22. S. Raza, G. Toscano, A.-P. Jauho, M. Wubs, and N. A. Mortensen, "Unusual resonances in nanoplasmonic structures due to nonlocal response," Phys. Rev. B 84, 121412(R) (2011).

23. C. David and F. J. García de Abajo, "Spatial nonlocality in the optical response of metal nanoparticles," J. Phys. Chem. C 115, 19470-19475 (2011).

24. N. A. Mortensen, S. Raza, M. Wubs, T. Søndergaard, and S. I. Bozhevolnyi, "A generalized non-local optical response theory for plasmonic nanostructures," Nat. Commun. 5, 3809 (2014).

25. S. Raza, S. I. Bozhevolnyi, M. Wubs, and N. A. Mortensen, "Nonlocal optical response in metallic nanostructures," J. Phys. Cond. Matter. 27, 183204 (2015).

26. C. David, J. Christensen, and N. A. Mortensen, "Spatial dispersion in two-dimensional plasmonic crystals: Large blueshifts promoted by diffraction anomalies," Phys. Rev. B 94, 165410 (2016).

27. V. V. Kresin, "Collective resonances and response properties of elec- trons in metal clusters," Phys. Rep. 220, 1 (1992).

28. M. Brack, "The physics of simple metal clusters: self-consistent jellium model and semiclassical approaches," Rev. of Mod. Phys. 65, 667 (1993).

29. M. Brack, "Multipole vibration of small alkali-metal spheres in a semiclassical description," Phys. Rev. B 39, 3533 (1989).

30. J. Jacak, J. Krasnyj, W. Jacak, R. Gonczarek, A. Chepok, and L. Jacak, "Surface and volume plasmons in metallic nanospheres in semiclassical RPA-type approach; near-field coupling of surface plasmons with semiconductor substrate," Phys. Rev. B 82, 035418 (2010).

31. W. Ekardt, "Size-dependent photoabsorption and photoemission of small metal particles," Phys. Rev. B 31, 6360 (1985)

32. W. Ekardt, "Anomalous inelastic electron scattering from small metal particles," Phys. Rev. B 33, 8803 (1986).

33. D. Pines and D. Bohm, "A collective description of electron interactions: II. collective vs individual particle aspects of the interactions," Phys. Rev. 85, 338 (1952)

34. D. Pines and D. Bohm, "A collective description of electron interactions: III. Coulomb interactions in a degenerate electron gas," Phys. Rev. 92 609 (1953).

35. W. Jacak, J. Krasnyj, J. Jacak, R. Gonczarek, A. Chepok, L. Jacak, D. Hu, and D. Schaadt, "Radius dependent shift of surface plasmon frequency in large metallic nanospheres: theory and experiment," J. of Appl. Phys. 107, 124317 (2010).

36. W. Jacak, E. Popko, A. Henrykowski, E. Zielony, K. Gwozdz, G. Luka R. Pietruszka, B. Witkowski, L. Wachnicki, L.-B. Chang, and M.-J. Jeng, "On the size dependence and spatial range for the plasmon effect in photovoltaic efficiency enhancement," Solar Energy Materials and Solar Cells 147, 1 (2016).

37. W. Jacak, "Lorentz friction for surface plasmons in metallic nanospheres," Journal of Physical Chemistry C 119, 6749 (2015).

38. H. R. Stuart and D. G. Hall, "Enhanced dipole-dipole interaction between elementary radiators near a surface," Phys. Rev. Lett. 80, 5663 (1998).

39. K. Okamoto, I. Niki, A. Shvartser, Y. Narukawa, T. Mukai, and A. Scherer, "Surface plasmon enhanced spontaneous emission rate of' InGaN/GaN QW probed by time-resolved photolumimnescence spectroscopy," Nat. Mater. 3, 601 (2004)

40. C. Wen, K. Ishikawa, M. Kishima, and K. Yamada, "Effects of silver particles on the photovoltaic properties of dye-sensitized $\mathrm{TiO}_{2}$ thin films," Sol. Cells 61, 339 (2000).

41. F. Masia, W. Langbein, and P. Borri, "Measurement of the dynamics of plasmons inside individual gold nanoparticles using a femtosecond phase-resolved microscope," Phys. Rev. B 85, 235403 (2012).

42. T. L. Temple and D. M. Bagnall, "Optical properties of gold and aluminium nanoparticles for silicon solar cell applications," J. of Appl. Phys/ 109, 084343 (2011).

43. L. D. Landau and L. M. Lifshitz, Quantum mechanics. Nonrelativistic theory (Pergamon Press, Oxford, 1965).

44. M. L. Brongersma, J. W. Hartman, and H. A. Atwater, "Electromagnetic energy transfer and switching in nanoparticle chain arrays below the diffraction limit," Phys. Rev. B 62, R16356 (2000).

45. L. D. Landau and E. M. Lifshitz, Field Theory (Nauka, Moscow, 1973).

46. W. Jacak, J. Krasnyj, J. Jacak, W. Donderowicz, and L. Jacak, "Mechanism of plasmon mediated enhancement of PV efficiency," J. Phys. D: Appl. Phys. 44, 055301 (2011).

47. P. S. Kiriejew, Physics of Semiconductors (PWN, Warsaw, 1969).

48. L. Landau and E. Lifszyc, Electrodynamics of Continuous Media, vol. 8 of Course of Theoretical Physics (Pergamon Press, 1960).

49. D. E. Aspnes and A. A. Studna, "Dielectric functions and optical parameters of $\mathrm{Si}, \mathrm{Ge}, \mathrm{GaP}, \mathrm{GaAs}, \mathrm{GaSb}, \mathrm{InP}$, InAs, and InSb from 1.5 to 6.0 eV," Phys. Rev. B 27, 985-1009 (1983).

50. M. Jeng, Z. Chen, Y. Xiao, L. Chang, J. Ao, Y. Sun, E. Popko, W. Jacak, and L. Chow, "Improving efficiency of multicrystalline silicon and cigs solar cells by incorporating metal nanoparticles," Materials 8, 6761 (2015)

51. F. J. García de Abajo, "Nonlocal effects in the plasmons of strongly interacting nanoparticles, dimers, and waveguides," J. Phys. Chem. C 
112, 17983-17987 (2008).

52. E. Prodan, C. Radloff, N. J. Halas, and P. Nordlander, "A hybridization model for the plasmon response of complex nanostructures," Science 302, 419-422 (2003).

53. M. Danckwerts and L. Novotny, "Optical frequency mixing at coupled gold nanoparticles," Phys. Rev. Lett. 98, 026104 (2007).

54. P. S. Kumar, I. Pastoriza-Santos, B. Rodríguez-González, F. J. G. de Abajo, and L. M. Liz-Marzán, "High-yield synthesis and optical response of gold nanostars," Nanotechnology 19, 015606 (2008).

55. J. A. Scholl, A. L. Koh, and J. A. Dionne, "Quantum plasmon resonances of individual metallic nanoparticles," Nature 483, 421-427 (2012).

56. C. Ciracì, R. T. Hill, J. J. Mock, Y. Urzhumov, A. I. FernándezDomínguez, S. A. Maier, J. B. Pendry, A. Chilkoti, and D. R. Smith, "Probing the ultimate limits of plasmonic enhancement," Science 337, 1072-1074 (2012).

57. S. Raza, N. Stenger, S. Kadkhodazadeh, S. V. Fischer, N. Kostesha, A.-P. Jauho, A. Burrows, M. Wubs, and N. A. Mortensen, "Blueshift of the surface plasmon resonance in silver nanoparticles studied with EELS," Nanophotonics 2, 131 (2013).

58. H. Haberland, "Looking from both sides," Nature 494, E1-E2 (2013).

59. S. Raza, S. Kadkhodazadeh, T. Christensen, M. Di Vece, M. Wubs, N. A. Mortensen, and N. Stenger, "Multipole plasmons and their disappearance in few-nanometer silver nanoparticles," Nat. Commun. 6, 8788 (2015).

60. R. Ruppin, "Optical properties of spatially dispersive dielectric spheres," J. Opt. Soc. Am. 71, 755-758 (1981).

61. B. B. Dasgupta and R. Fuchs, "Polarizability of a small sphere including nonlocal effects," Phys. Rev. B 24, 554-561 (1981).

62. R. Fuchs and F. Claro, "Multipolar response of small metallic spheres: Nonlocal theory," Phys. Rev. B 35, 3722-3727 (1987).

63. U. Kreibig and M. Vollmer, Optical Properties of Metal Clusters, vol. 25 of Springer Series in Materials Science (Springer, Heidelberg, 1995).

64. R. Ruppin, "Extinction properties of thin metallic nanowires," Optics Communications 190, 205-209 (2001).

65. A. Eguiluz, S. C. Ying, and J. J. Quinn, "Influence of the electron density profile on surface plasmons in a hydrodynamic model," Phys. Rev. B 11, 2118-2121 (1975).

66. J. E. Sipe, V. C. Y. So, M. Fukui, and G. I. Stegeman, "Analysis of second-harmonic generation at metal surfaces," Phys. Rev. B 21, 43894402 (1980).

67. J. Aizpurua and A. Rivacoba, "Nonlocal effects in the plasmons of nanowires and nanocavities excited by fast electron beams," Phys. Rev. B 78, 035404 (2008).

68. J. M. McMahon, S. K. Gray, and G. C. Schatz, "Optical properties of nanowire dimers with a spatially nonlocal dielectric function," Nano Lett. 10, 3473-3481 (2010).

69. A. Wiener, A. I. Fernández-Domínguez, A. P. Horsfield, J. B. Pendry, and S. A. Maier, "Nonlocal effects in the nanofocusing performance of plasmonic tips," Nano Lett. 12, 3308-3314 (2012).

70. D. de Ceglia, S. Campione, M. A. Vincenti, F. Capolino, and M. Scalora, "Low-damping epsilon-near-zero slabs: Nonlinear and nonlocal optical properties," Phys. Rev. B 87, 155140 (2013).

71. C. David, N. A. Mortensen, and J. Christensen, "Perfect imaging, epsilon-near zero phenomena and waveguiding in the scope of nonlocal effects," Sci. Rep. 3, 2526 (2013).

72. Y. Luo, A. I. Fernandez-Dominguez, A. Wiener, S. A. Maier, and J. B. Pendry, "Surface plasmons and nonlocality: A simple model," Phys. Rev. Lett. 111, 093901 (2013).

73. T. Christensen, W. Yan, S. Raza, A.-P. Jauho, N. A. Mortensen, and M. Wubs, "Nonlocal response of metallic nanospheres probed by light, electrons, and atoms," ACS Nano 8, 1745-1758 (2014).

74. K. Kluczyk and W. Jacak, "Damping-induced size effect in surface plasmon resonance in metallic nano-particles: Comparison of rpa microscopic model with numerical finite element simulation (comsol) and mie approach," Journal of Quantitative Spectroscopy and Radiative Transfer 168, 78-88 (2016).

75. C. David and F. J. García de Abajo, "Surface plasmon dependence on the electron density profile at metal surfaces," ACS Nano 8, 9558-9566 (2014).

76. N. D. Lang and W. Kohn, "Theory of metal surfaces: Charge density and surface energy," Phys. Rev. B 1, 4555-4568 (1970).

77. F. J. G. de Abajo, "Multiple scattering of radiation in clusters of dielectrics," Phys. Rev. B 60, 6086-6102 (1999).

78. F. E. Low, Classical Field Theory - Electromagnetism and Gravitation (Wiley-VCH Verlag, New York, 1997).

79. J. D. Jackson, Classical Electrodynamics (John Wiley \& Sons, Inc., New York, 1976). 